\title{
Las relaciones interpersonales en el desempeño laboral docente
}

\author{
Mg. Nancy Aracely Castro Ramírez \\ P7001249979@ucvvirtual.edu.pe \\ ORCID: 0000-0003-3157-6320 \\ Universidad César Vallejo
}

Mg. Wendy Yesenia Alvarado Torres P7001249969@ucvvirtual.edu.pe ORCID: 0000-0002-8133-8577 Universidad César Vallejo

Mg. Johanna del Carmen Romero Jiménez P7001254326@ucvvirtual.edu.pe ORCID: 0000-0001-8733-8191 Universidad César Vallejo

\section{Mg. David Armando Mondragón Sánchez} damondragonm@ucvvirtual.edu.pe ORCID: 0000-0002-2025-3377 Universidad César Vallejo

\section{RESUMEN}

Piura - Perú

La presente investigación tuvo como objetivo general diseñar un programa de estrategias basadas en relaciones interpersonales para optimizar el desempeño laboral docente en la Unidad Educativa Juan Bautista Aguirre, Distrito Daule 2020. Se empleó el enfoque cuantitativo, tipo aplicada, diseño no experimental, transversal, descriptivo; para la recolección de los datos se utilizó la encuesta y el cuestionario con 24 interrogantes aplicado a 72 docentes de las tres jornadas ordinarias, luego se procesaron en Microsoft Excel y para el análisis se empleó el programa SPSS versión 26. Los datos de las tablas evidencian que las relaciones interpersonales no son apropiadas y ello afecta el desempeño laboral docente. Los resultados relacionados a la variable relaciones interpersonales, los docentes calificaron el 78\% deficiente, el 19\% regular y el 3\% bueno; se aprecia que los docentes no se relacionan fácilmente y ello contribuye al surgimiento de complicaciones en el desarrollo de sus actividades laborales; en torno a la variable desempeño laboral docente el 69\% califica nivel deficiente, el 28\% regular y el $3 \%$ bueno, consideran que el desempeño laboral de los docentes en las diferentes actividades pedagógicas no es óptimo.

Palabras clave: relaciones interpersonales; desempeño laboral docente; estrategias. 


\title{
Interpersonal relationships in teacher job performance
}

\begin{abstract}
The general objective of this research was to design a program of strategies based on interpersonal relationships to optimize teacher work performance in the Juan Bautista Aguirre Educational Unit, Daule 2020 District. The quantitative approach was used, applied type, non-experimental, cross-sectional, descriptive design; For data collection, the survey and questionnaire with 24 questions applied to 72 teachers from the three ordinary days were used, then it was processed in Microsoft Excel and the SPSS version 26 program was used for the analysis. The data in the tables show that interpersonal relationships are not appropriate and this affects teacher job performance. The results related to the variable interpersonal relationships, teachers rated $78 \%$ poor, $19 \%$ fair and $3 \%$ good; It is appreciated that teachers do not relate easily and this contributes to the emergence of complications in the development of their work activities; Regarding the variable teacher job performance, $69 \%$ rate a poor level, $28 \%$ regular and $3 \%$ good, they consider that the job performance of teachers in the different pedagogical activities is not optimal.
\end{abstract}

Keywords: interpersonal relations; teaching labor performance; strategies.

Artículo recibido: 18. Julio. 2021

Aceptado para publicación: 08. Agosto. 2021 Correspondencia: P7001249979@ucvvirtual.edu.pe Conflictos de Interés: Ninguna que declarar 


\section{INTRODUCCIÓN}

Las relaciones interpersonales hoy en día están presentes en todas las actividades que desarrolla el ser humano, son significativas y permiten relacionarse en todo tipo de contextos. Velmurugan (2016) señala que son asociaciones que surgen entre las personas y pueden ser efímeras o perdurar con el tiempo. Obakpolo (2015) resalta que las buenas relaciones interpersonales son de gran ayuda en el ámbito personal e institucional. Lacunza \& Contini (2016) señalan que cuando existe relaciones positivas entre los individuos se fomenta el disfrute y eficiencia en cualquier actividad.

En todo el mundo las personas están inmersas en contextos sean estos sociales, familiares, educativos, laborales entre otros; la convivencia y las relaciones interpersonales entre ellos son primordiales e importantes para el sano vivir. Sin embargo no es tarea simple convivir con los demás, suelen aflorar complicaciones como la falta de comunicación, poca interacción, desinterés, envidia, contiendas y ello obstaculiza las relaciones. Vera, López, Valle \& Mazacón (2017) enfatizan que el comportamiento de las personas depende del momento, del contexto y por supuesto de las personas; además, señalan que las relaciones interpersonales han proliferado en complejidad. Calcina (2014) señaló que los profesores que encuentran dificultades en su lugar de trabajo no se desenvuelven adecuadamente. Szostek (2019) determina que cuando las relaciones entre los empleados son de baja calidad desfavorecen las actividades de las instituciones.

En el contexto educativo las relaciones interpersonales entre los docentes ocupan un lugar primordial, en él se desarrollan ejes como la comunicación, interacción, motivación, convivencia que sin duda son esenciales para que el desempeño laboral de los educadores fluya con normalidad. López, Efstathios, Herrera \& Apolo (2018) señalan que cuando el clima laboral de los profesionales de educación es acogedor, entonces sus prácticas se desarrollan en ambientes exitosos.

El desempeño laboral del docente alude a su práctica profesional y si bien es cierto que los conocimientos que poseen y comparten con sus estudiantes son importantes en el proceso de enseñanza aprendizaje, las relaciones interpersonales entre ellos ayudan en su desempeño. Castaño (2014) determinó que es fundamental la sana convivencia escolar y que se debe evitar solamente centrarse en la parte pedagógica, al contrario el potencial de cada maestro se puede aprovechar en actividades de carácter social y que ayuden al logro de las metas institucionales. 
La motivación juega un papel preponderante en el desempeño de los docentes, así como las relaciones interpersonales. Parada (2017) acentúa, para que el desempeño docente sea apropiado debe existir por parte de los directivos una buena motivación, así logran alcanzar objetivos comunes. De Asis Galvez (2018) resalta que en el comportamiento de toda persona la motivación es importante, constituye un componente esencial y los dirige al éxito.

El estudio aborda las relaciones interpersonales en el Desempeño Laboral Docente en la Unidad Educativa Juan Bautista Aguirre, Daule 2020; la problemática que viven los docentes perjudica las relaciones y por ende el desempeño laboral, en torno a ello, el problema central del estudio es: ¿Cuál es el diseño que deberá tener un programa de estrategias basadas en relaciones interpersonales con el propósito de optimizar el desempeño laboral docente en la Unidad Educativa Juan Bautista Aguirre, del distrito Daule 2020?

El estudio se justifica puesto que las relaciones interpersonales son esenciales en el desempeño laboral de todo empleado y más aún en los docentes que interactúan a diario con todos los actores de la comunidad educativa, es así que la contribución de un programa de estrategias basadas en relaciones interpersonales al fortalecimiento de la práctica de los docentes, coadyuva en la interacción de los profesores, incrementa la buena comunicación, participen en todas las actividades educativas, asistan con entusiasmo al lugar de trabajo y acudan a las aulas motivados.

Como hipótesis general se planteó: diseño de un programa de estrategias basadas en relaciones interpersonales con el propósito de optimizar el desempeño laboral docente en la Unidad Educativa Juan Bautista Aguirre, Distrito Daule 2020.

El objetivo general del estudio es: proponer un programa de estrategias basadas en relaciones interpersonales para optimizar el desempeño laboral docente en la Unidad Educativa Juan Bautista Aguirre, Distrito Daule 2020. Los objetivos específicos que se plantean son: primero: diagnosticar las características que presentan las relaciones interpersonales de los docentes de la Unidad Educativa Juan Bautista Aguirre, Distrito Daule 2020; segundo: identificar los factores asociados al desempeño laboral de los docentes de la Unidad Educativa Juan Bautista Aguirre, Distrito Daule 2020; tercero diseñar un programa de estrategias de relaciones interpersonales para optimizar el desempeño laboral docente de la Unidad Educativa Juan Bautista Aguirre, Distrito Daule 
2020 y el cuarto validar el programa de estrategias basadas en las relaciones interpersonales para optimizar el desempeño laboral docente de la Unidad Educativa Juan Bautista Aguirre, Distrito Daule 2020.

\section{ESTRATEGIAS METODOLÓGICAS O MATERIALES Y MÉTODOS}

\section{Enfoque de investigación}

El actual estudio tiene un enfoque cuantitativo, al respecto Jameel, Shaheen \& Majid (2018) mencionan que entre las principales características de estos estudios es que la medición de los datos que se recopilan es exacta y para el recojo de los mismos se puede emplear la encuesta con anticipación.

\section{Tipo de investigación}

En cuanto al tipo es aplicada, pues busca contribuir al mejoramiento de las relaciones interpersonales en el desempeño laboral de los docentes. Naupas, Valdivia, Palacios \& Romero (2018) aluden en que estas investigaciones contribuyen en la solución de problemas de tipo social en cualquier comunidad.

\section{Diseño de investigación}

El diseño es no experimental, transversal, descriptivo. Primero se indaga las variables que intervienen, luego se utiliza la técnica para la recopilación de los datos por única vez y finalmente se describen características específicas; al respecto Naupas et al. (2018) señalan que el propósito de estas investigaciones es agrupar datos de los sujetos que intervienen en el estudio.

\section{Población y muestra}

Población: De acuerdo a Majid (2018) la población objetivo es la de interés para el investigador, es decir en ellos se va a realizar un estudio y obtener información, sostiene que es conveniente trabajar con grupos reducidos. La población estuvo conformada por 72 docentes de los niveles de básica superior y bachillerato de las tres jornadas ordinarias de la Unidad Educativa Juan Bautista Aguirre, Distrito 09D19.

Muestra: La población inmersa en la investigación es limitada por esta razón se trabajó con el $100 \%$ de los docentes, es decir la muestra empleada fue censal. Arias, Villasís \& Miranda (2016) enfatizan que cuando el número de participantes es pequeño se ahorra tiempo y recursos.

Técnicas e instrumentos de recolección de datos.

Técnica 
Para obtener información certera se empleó como técnica la encuesta. López \& Fachelli (2015), sustentan que esta recoge datos de los involucrados permitiendo obtener información detallada del problema objeto de investigación.

\section{Instrumento}

Granados Muñoz (2020) precisa que el cuestionario es una herramienta metodológica utilizada para la recolección de datos y no es necesario la presencia del investigador. El instrumento utilizado fue el cuestionario, en él intervinieron 24 interrogantes que involucra las variables intervinientes, así como las dimensiones e indicadores.

\section{Procedimientos}

Fueron varios los procesos metodológicos empleados. Inicialmente se solicitó el permiso para la aplicación del instrumento a la autoridad respectiva, concedido el permiso se envió el consentimiento informado a los docentes por medio de un formulario en Google Forms, el mismo contiene el cuestionario, es decir aceptado el consentimiento da paso al desarrollo del cuestionario. Los datos fueron procesados en Microsoft Excel y para el análisis estadístico se empleó el programa SPPP versión 26.

\section{Análisis de los datos}

Las tablas de frecuencias elaboradas en Microsoft Excel permitieron obtener una visión clara de los datos y proceder en su análisis. En torno a la verificación de normalidad de la distribución de los datos se aplicó la prueba de Kolmogorov-Smirnov, pues el tamaño de la muestra fue 72 docentes.

\section{RESULTADOS Y DISCUSIÓN}

Se presentan los datos obtenidos en el cuestionario aplicado a 72 docentes de la Unidad Educativa Juan Bautista Aguirre, Distrito 09D19.

\section{Tabla 1}

Nivel de relaciones interpersonales

\begin{tabular}{lcc} 
& \multirow{2}{*}{ Nivel } & \multicolumn{2}{c}{ Docentes } \\
\cline { 2 - 3 } & $\mathbf{N}^{\circ}$ & $\mathbf{\%}$ \\
\hline Deficiente & 56 & $78 \%$ \\
Regular & 14 & $19 \%$ \\
Bueno & 2 & $3 \%$ \\
\hline Total & $\mathbf{7 2}$ & $\mathbf{1 0 0 \%}$ \\
\hline
\end{tabular}

Nota. Instrumentos de investigación. Elaborado por: Nancy Aracely Castro Ramírez.

\section{Interpretación:}


La tabla 1 muestra el nivel de las relaciones interpersonales en los docentes: el $78 \%$ califica nivel deficiente, consideran que las relaciones interpersonales no son afectuosas y que alteran directa e indirectamente el normal desenvolvimiento de las actividades de los docentes; el 19\% califica nivel regular, referencian que las relaciones interpersonales son algo afectuosas y el 3\% califica nivel bueno, referencian que las relaciones son afectuosas.

\section{Tabla 2}

Nivel de desempeño laboral docente

\begin{tabular}{lcc}
\hline & Nivel & \multicolumn{2}{c}{ Docentes } \\
\cline { 2 - 3 } & $\mathbf{N}^{\circ}$ & $\%$ \\
\hline Deficiente & 50 & $69 \%$ \\
Regular & 20 & $28 \%$ \\
Bueno & 2 & $3 \%$ \\
\hline Total & $\mathbf{7 2}$ & $\mathbf{1 0 0 \%}$ \\
\hline
\end{tabular}

Nota. Instrumentos de investigación. Elaborado por: Nancy Aracely Castro Ramírez.

\section{Interpretación:}

La tabla 2 muestra el análisis del desempeño laboral docente: el 69\% califica nivel deficiente, consideran que el desempeño laboral de los docentes en las diferentes actividades pedagógicas no es óptimo; el 28\% califica nivel regular, consideran que el desempeño laboral docente en ciertas actividades es óptimo y el 3\% califica nivel bueno, consideran que el desempeño laboral es óptimo.

\section{Tabla 3}

Nivel de dimensión de comunicación

\begin{tabular}{lcc}
\hline & Nivel & \multicolumn{2}{c}{ Docentes } \\
\cline { 2 - 3 } & $\mathbf{N}^{\circ}$ & $\mathbf{\%}$ \\
\hline Deficiente & 44 & $61 \%$ \\
Regular & 21 & $29 \%$ \\
Bueno & 7 & $10 \%$ \\
\hline Total & $\mathbf{7 2}$ & $\mathbf{1 0 0 \%}$ \\
\hline
\end{tabular}

Nota. Instrumentos de investigación. Elaborado por: Nancy Aracely Castro Ramírez.

\section{Interpretación:}


La tabla 3 muestra los datos concernientes a la dimensión comunicación, la misma refleja que el $61 \%$ de los docentes calificaron nivel deficiente, refieren que la comunicación entre ellos no es oportuna; el 29\% calificaron nivel regular, refieren en que hay momentos en que sí se comunican oportunamente y el $10 \%$ calificaron nivel bueno, ellos refieren en que la comunicación sí es oportuna.

\section{Tabla 4}

Nivel de dimensión asertividad

\begin{tabular}{lcc} 
& Nivel & \multicolumn{2}{c}{ Docentes } \\
\cline { 2 - 3 } & $\mathbf{N}^{\circ}$ & \% \\
\hline Deficiente & 56 & $78 \%$ \\
Regular & 13 & $18 \%$ \\
Bueno & 3 & $4 \%$ \\
Total & $\mathbf{7 2}$ & $\mathbf{1 0 0 \%}$ \\
\hline
\end{tabular}

Nota. Instrumentos de investigación. Elaborado por: Nancy Aracely Castro Ramírez.

\section{Interpretación:}

La tabla 4 muestra datos concernientes a la dimensión asertividad, el 78\% calificó nivel deficiente, sostienen que los docentes no son asertivos; el $18 \%$ califica nivel regular, sostienen que es necesario que se desarrolle esta característica en los docentes y el $4 \%$ calificó nivel bueno, sostienen que son asertivos los docentes.

\section{Tabla 5}

Nivel de dimensión interacción

\begin{tabular}{lcc}
\hline \multirow{2}{*}{ Nivel } & \multicolumn{2}{c}{ Docentes } \\
\cline { 2 - 3 } & $\mathbf{N}^{\circ}$ & $\mathbf{\%}$ \\
\hline Deficiente & 57 & $79 \%$ \\
Regular & 12 & $17 \%$ \\
Bueno & 3 & $4 \%$ \\
Total & $\mathbf{7 2}$ & $\mathbf{1 0 0 \%}$
\end{tabular}

Nota. Instrumentos de investigación. Elaborado por: Nancy Aracely Castro Ramírez.

\section{Interpretación:}

La tabla 5 refiere a la dimensión interacción, el 79\% calificó nivel deficiente, manifiestan que en el desarrollo de actividades pedagógicas los docentes no interactúan, es decir no trabajan de manera colaborativa; el 17\% calificó nivel regular, manifiestan que hay ciertos 
docentes que interactúan en actividades especialmente en las planificaciones y $4 \%$ calificó nivel bueno, los resultados responden a que los docentes sí interactúan en todas las actividades.

\section{Tabla 6}

Nivel de dimensión motivación laboral

\begin{tabular}{lcc} 
& Nivel & \multicolumn{2}{c}{ Docentes } \\
\cline { 2 - 3 } & $\mathbf{N}^{\circ}$ & $\mathbf{\%}$ \\
\hline Deficiente & 45 & $63 \%$ \\
Regular & 25 & $35 \%$ \\
Bueno & 2 & $3 \%$ \\
Total & $\mathbf{7 2}$ & $\mathbf{1 0 0 \%}$ \\
\hline
\end{tabular}

Nota. Instrumentos de investigación. Elaborado por: Nancy Aracely Castro Ramírez.

\section{Interpretación:}

La tabla 6 exhibe datos relacionados a la dimensión motivación laboral. El 63\% de los docentes calificó nivel deficiente, consideran que no hay motivación especialmente porque la remuneración que perciben no representa el trabajo realizado, de igual manera sostienen que las autoridades no son mediadores en este proceso; el 35\% calificó nivel regular, consideran que en ciertas ocasiones hay estímulos hacia los docentes y el 3\% calificó nivel bueno, estiman que la remuneración y los procesos pedagógicos que comparten con sus compañeros son motivados principalmente por las autoridades y la remuneración que perciben es la justa.

\section{Tabla 7}

Nivel de dimensión trabajo en equipo

\begin{tabular}{lcc}
\hline & \multicolumn{2}{c}{ Docentes } \\
\cline { 2 - 3 } & $\mathbf{N}^{\circ}$ & \% \\
\hline Deficiente & 49 & $68 \%$ \\
Regular & 17 & $24 \%$ \\
Bueno & 6 & $8 \%$ \\
Total & $\mathbf{7 2}$ & $\mathbf{1 0 0 \%}$
\end{tabular}

Nota. Instrumentos de investigación. Elaborado por: Nancy Aracely Castro Ramírez.

\section{Interpretación:}


La tabla 7 evidencia datos referentes a la dimensión trabajo en equipo. El 68\% calificó nivel deficiente, aseguran que las actividades en la institución no se trabajan en equipo; el 24\% calificó nivel regular, consideran que hay ciertas actividades escolares en las que participan todos los docentes y el $8 \%$ calificó nivel bueno, aseguran que el trabajo en equipo ha ayudado al cumplimiento de los objetivos institucionales.

En torno a la distribución de los datos se utilizó la prueba de Kolmogórov-Smirnov, se exhiben los siguientes datos:

\section{Tabla 8}

Prueba de normalidad de Kolmogórov-Smirnov de las variables relaciones interpersonales y desempeño laboral docente.

\begin{tabular}{lcc}
\hline & \multicolumn{2}{c}{ Kolmogorov-Smirnov } \\
\hline Prueba de normalidad & $\begin{array}{c}\text { Relaciones } \\
\text { interpersonales }\end{array}$ & $\begin{array}{c}\text { Desempeño } \\
\text { laboral docente }\end{array}$ \\
\hline $\mathrm{N}$ & 72 & 72 \\
\hline Test Statistic & 0,173 & 0,180 \\
\hline Asymp. Sig. (2-tailed) & $0,000^{\mathrm{c}}$ & $0,000^{\mathrm{c}}$ \\
\hline
\end{tabular}

Nota. Reporte SPSS. Elaborado por: Nancy Aracely Castro Ramírez.

\section{Interpretación:}

La tabla 8 muestra la distribución de los datos entre las variables relaciones interpersonales y desempeño laboral docente, los resultados no son normales pues el nivel de significancia fue de 0.000 menor a 0.05 por ello fue necesario la aplicación de pruebas no paramétricas con el fin de aceptar la hipótesis de la investigación. Chi-Cuadrado fue utilizada para someter a prueba la hipótesis.

\section{Tabla 9}

Prueba de Chi cuadrado.

\begin{tabular}{lcc}
\hline Prueba no paramétrica & $\begin{array}{c}\text { Relaciones } \\
\text { interpersonales }\end{array}$ & $\begin{array}{c}\text { Desempeño } \\
\text { Laboral docente }\end{array}$ \\
\hline Chi-Square & $12,028^{\mathrm{a}}$ & $11,000^{\mathrm{b}}$ \\
\hline df & 18 & 12 \\
\hline Asymp. Sig. & 0,000 & 0,000 \\
\hline
\end{tabular}

Nota. Reporte SPSS. Elaborado por: Nancy Aracely Castro Ramírez.

\section{CONCLUSIÓN O CONSIDERACIONES FINALES}


Primero.- Los docentes de la Unidad Educativa Juan Bautista Aguirre deben incorporar estrategias basadas en relaciones interpersonales y así mejorar el desempeño laboral docente.

Segundo.- Las características que influyen en las malas relaciones interpersonales son: falta de confianza, no expresan su forma de pensar, no intercambian conocimientos y experiencias, poca comunicación, no son asertivos.

Tercero.- La motivación, responsabilidad, liderazgo efectivo, ambiente laboral, compensación, reconocimiento, trabajo en equipo e incluso el desarrollo profesional son factores que no se desarrollan en un alto nivel en los docentes de la Unidad Educativa Juan Bautista Aguirre.

\section{LISTA DE REFERENCIAS}

Arias, J., Villasís, M. \& Miranda, M. (2016). El protocolo de Investigación III: la Población de Estudio. Alergia México, 63(2), 201-206. Recuperado de http://www.redalyc.org/articulo.oa?id=486755023011

Calcina, Y. (2014). El clima institucional y su incidencia en el desempeño laboral de los docentes de la facultad de ciencias sociales de la Universidad Nacional del altiplano y facultad de ciencias de la educación Universidad Andina Néstor Cáceres Velásquez - Perú 2012. Scielo, 5(1), 22-29. Recuperado de http://www.scielo.org.pe/scielo.php?script=sci_arttext\&pid=S221971682014000100003

Castaño, R. (2014). Estrategias de gestión para el mejoramiento de relaciones interpersonales entre iguales de los estudiantes del curso 702 jornada de la tarde del colegio Atenas I.E.D. Universidad Libre. Colombia.

De Asis, G. (2018). Work Motivation and Awards Received, and Pupils' Academic Performance in Elementary Schools in the Division of Northern Samar. International Research Conference on Higher Education, 522-539. Recuperado de https://doi.org/10.18502/kss.v3i6.2402

Granados, R. (2020). Revisión teórica de herramientas metodológicas aplicadas en la investigación criminológica. Derecho Y Cambio Social, (59), 501-511. Recuperado de https://www.researchgate.net/publication/342329674_Revision_teorica_de_herr amientas_metodologicas_aplicadas_en_la_investigacion_criminologica 
Jameel, B., Shaheen, S. \& Majid, U. (2018). Introduction to Qualitative Research for Novice Investigators. URNCST Journal 2(6), 1-12. Recuperado de https://doi.org/10.26685/urncst.57

Lacunza, A. \& Contini, E. (2016). Relaciones interpersonales positivas: los adolescentes como protagonistas. Psicodebate, 16(2), 73-94. doi: http://dx.doi.org/10.18682/pd.v16i2.598

López, M., Efstathios, S., Herrera, M. \& Apolo, D. (2018). Clima escolar y desempeño docente: un caso de éxito. Aproximaciones a escuelas públicos de la provincia de Carchi-Ecuador. Espacios, 39(35), 5. Recuperado de https://www.revistaespacios.com/a18v39n35/a18v39n35p05.pdf

López, P. \& Fachelli, S. (2015). Metodología de la Investigación Social Cuantitativa. Recuperado de https://ddd.uab.cat/pub/caplli/2016/163564/metinvsoccua_a2016_cap1-2.pdf

Majid, U. (2018). Research Fundamentals: Study Design, Population, and Sample Size. URNCST Journal, 2(1), 1-7. doi: https://doi.org/10.26685/urncst.16

Ñaupas, H., Valdivia, M., Palacios, J. \& Romero, H. (2018). Metodología de la Investigación. Cuantitativa-Cualitativa y Redacción de la Tesis. Recuperado de https://corladancash.com/wp-content/uploads/2020/01/Metodologia-de-la-invcuanti-y-cuali-Humberto-Naupas-Paitan.pdf

Obakpolo, P. (2015). Improving Interpersonal Relationship in Workplaces. IOSR Journal of Research \& Method in Education, 5(6), 115-125. Recuperado de https://www.iosrjournals.org/iosr-jrme/papers/Vol-5\%20Issue-6/Version2/P0562115125.pdf

Parada, J. (2017). Estrategias gerenciales para el reconocimiento del desempeño laboral docente. Mundo Fesc, 7(14), 42-56. Recuperado de https://www.fesc.edu.co/Revistas/OJS/index.php/mundofesc/article/view/140/19 8

Szostek, D. (2019). The Impact of the Quality of Interpersonal Relationships between Employees on Counterproductive Work Behavior: A Study of Employees in Poland. Sustainability, 11(21), 1-33. doi: 10.3390/su11215916 
Velmurugan, C. (2016). Interpersonal Relationship and Organizational Effectiveness. International Journal of Business Management and Leadership, 7(1), 1-5. Recuperado de https://www.ripublication.com/ijbml16/ijbmlv7n1_01.pdf

Vera, M., López, F., Valle, V. \& Mazacón, M. (2017). Habilidades Sociales. Salud y Vida, 1(2), $\quad$ 08-15. Recuperado de https://revistas.utb.edu.ec/index.php/saludyciencias/article/view/424 